\title{
Psychological and Neurobiological Precursors of Alcohol Use Disorders in High-Risk Youth
}

\author{
Shirley Y. Hill ${ }^{1,2,3}$ • Jessica O'Brien ${ }^{2}$ \\ Published online: 8 April 2015 \\ (C) Springer International Publishing AG 2015
}

\section{Introduction}

Alcohol use disorders (AUDs) are a major public health problem in the USA and many other parts of the world. Data from the National Epidemiologic Survey on Alcohol and Related Conditions indicates that the lifetime prevalence of AUDs is $30.3 \%$ in the USA [1]. Alcohol use disorders run in families, and a recent meta-analysis of twin and adoption studies indicates that the heritability for AUD is estimated to be 0.52 for men and 0.44 for women [2]. However, determining the specific genetic underpinnings of this disorder has been challenging given the multiple clinical subtypes of AUD and variable expression across the lifespan. AUD and related substance use disorder (SUD) phenotypes are thought to be controlled by multiple genes. Accordingly, the clinical heterogeneity seen among these phenotypes may reduce the statistical power to uncover significant associations between these genetic variants and the AUD phenotype. Consequently, increased attention has been focused on finding biological variation associated with these phenotypes that can be used to identify genes conferring increased susceptibility to the disorder. [3].

There has been increased interest in the study of endophenotypes, sometimes referred to as intermediate phenotypes, because it is believed that these indicators of pathology will be more proximal to genetic variation that may segregate with disease. The ideal endophenotype is one which exhibits heritability, is present in individuals displaying the pathology, is manifest in an individual whether or not illness is active, and is found in unaffected biological relatives of those who have the disorder at a higher rate than in the general population [4]. Many genes show pleiotropic effects with differing manifestations across the life span. Some AUD-associated genes appear to have behavioral and biological manifestations in childhood and adolescence that differ from those seen in adulthood. As one example, conduct disorder of adolescence has frequently been linked to substance use disorder outcome [5]. The 
identification of endophenotypes that emerge before the onset of alcohol use disorders has important implications for determining risk factors for AUD useful in targeted prevention and intervention efforts.

Some studies examining endophenotypes associated with AUD and related disorders have contrasted family history positive and negative offspring, with family history being defined by having an alcohol-dependent first-degree relative. Others, known as multiplex family designs, have utilized families at ultra-high risk for developing alcohol dependence through the presence of multiple relatives with alcohol dependence. The identification of biological and psychological characteristics of high- versus low-risk offspring has led to the discovery of both biological and psychological endophenotypes associated with AUD and related SUD. Longitudinal studies that follow high-risk offspring from childhood through adolescence and into young adulthood have identified potential biomarkers that contribute to risk and resilience within this population. This article will review recent advances in the search for biological and psychological endophenotypes associated with SUD, including findings of abnormal morphology of brain regions associated with executive control and affective regulation, atypical developmental trajectories of electrophysiological activity and postural control, and neurocognitive deficits in decision making and social cognition in high-risk offspring.

The majority of work done in an attempt to find the neurological underpinnings of psychiatric disorders including alcohol use disorders and related substance use disorders has focused on specific brain regions that may influence cognitive, social, and emotional aspects of an individual's functioning. Accordingly, this review will first focus on brain morphology of specific regions as they relate to risk for substance use disorders and then briefly discuss how the coordinated functioning of multiple brain regions can influence risk and resilience to these disorders.

\section{Adolescent Brain Development}

Marked changes in brain structure and function occur during adolescence, supporting development of cognitive, social, and emotional behavior [6]. The onset of adolescence is usually characterized by the start of pubertal maturation, which begins between the ages of 9 and 12 years, and typically starts 12 years earlier in female than in male individuals. The end of adolescence has less clear biological boundaries, and may extend into the early 20 s as individuals continue to experience changes in social roles and responsibilities [7]. Longitudinal research on brain morphology indicates that, throughout the brain, cortical white matter increases with age during childhood and adolescence, whereas cortical gray matter follows an
inverted-U shape over development, with regional volumes peaking at different ages $[8,9,10 \bullet]$. Subcortical brain regions show both linear and nonlinear changes with the caudate and putamen linearly decreasing in volume throughout adolescence, while the amygdala and hippocampus show an increase in size at the onset of puberty after which growth stabilizes [11].

There is substantial evidence from twin and family studies that the volume of specific brain structures is heritable [12, 13]. The influence of genes on human brain volume is already present in childhood, and variation in brain volumes remains largely explained by genetic factors, even in old age [13]. This suggests that offspring from families where alcohol dependence has a strong recurrence risk over multiple generations would be likely to have altered brain morphology that might predispose them to greater risk for developing SUD. Indeed, morphological brain changes during adolescence and young adulthood appear to vary by familial risk group status [3]. The following sections focus on familial risk group differences in morphology of the orbitofrontal cortex (OFC), amygdala, hippocampus, cerebellum, and brain networks associated with AUD/SUD risk.

\section{Morphological Characteristics of High-Risk Offspring}

Orbitofrontal Cortex The orbitofrontal cortex (OFC) is a prefrontal brain region implicated in addiction due to its association with inhibitory control and goal-directed behavior [14]. Adolescent and young adult offspring from multiplex, alcohol-dependent families have reduced volumes of the right orbitofrontal cortex [15, 16]. Furthermore, OFC white matter volume is significantly associated with lower scores on the Control scale of the Multidimensional Personality Questionnaire (MPQ), indicating that individuals with reduced OFC white matter volume show greater impulsivity [16]. Because personal exposure to drugs or alcohol could have neurotoxic effects on the OFC, analyses were performed removing those with a substance use disorder prior to the scan. These analyses continued to show risk group differences indicating that the findings were not the result of alcohol or drug exposures. The laterality of these findings in high-risk offspring $[15,16]$ appears to be in accord with previous observations of patients with unilateral lesions to the right or left OFC showing that right OFC lesions are uniquely associated with severe deficits in social and emotional processes [17]. Lesions to the right, but not left, OFC are associated with impaired performance on the Iowa Gambling Task (IGT), a measure of decision making that simulates a real-world decision situation requiring evaluation of the magnitude and timing of rewards and punishments under uncertain conditions [17, 18]. A 
Fig. 1 A significant relationship $(r=0.336, p=0.001)$ between total IGT scores and medial OFC volume is shown indicating that poorer IGT performance is associated with lesser medial OFC volume
Medial OFC Volume and IGT Performance

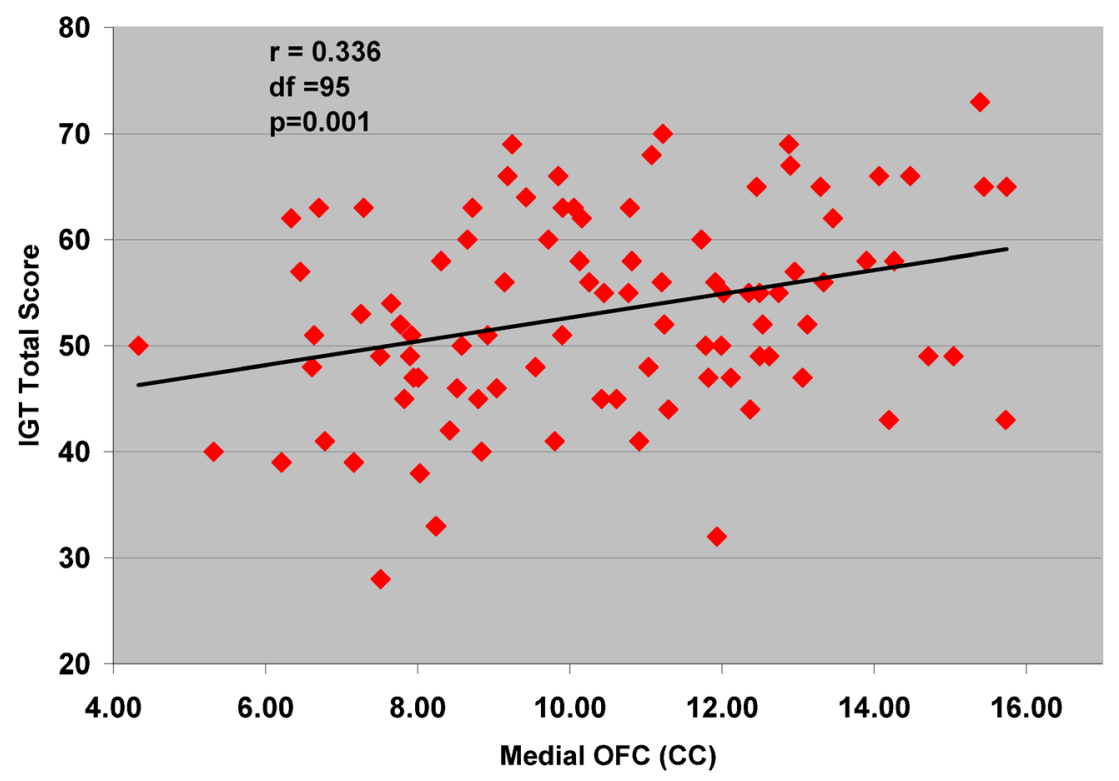

relationship between OFC volume and IGT performance has also been found in our lab (Hill et al., unpublished data). As may be seen in Fig. 1, across both high and lowrisk subjects, the relationship appears to be robust $(r=0.30)$. The origin of these deficits in behavioral control and decision-making processes may be related to developmental changes in OFC volume. Regression analysis of OFC volume with age shows highrisk male subjects deficient in volume relative to control male subjects though the resulting curves indicated that the high-risk male subjects did eventually catch up with the control male subjects [16].

Amygdala Drugs of abuse are associated with increased extracellular dopamine in the mesocorticolimbic system, including the amygdala, which may explain why the amygdala shows greater activation in response to drugrelated than neutral cues [19]. Adolescents and young adults from multiplex, alcohol-dependent families have been shown to have reduced volume of the right amygdala $[15,20,21 \bullet, 22]$. These results were observed in samples where either the majority of cases had not yet developed a substance use disorder [20] at the time of the scans, were alcohol naive [22], or the reduction in volume was seen even when substance use disorder cases were removed $[21 \bullet \bullet]$. Reduced amygdala volumes have also been documented in unaffected first-degree adult relatives of individuals with AUD compared to unaffected individuals with no family history [23••]. Furthermore, amygdala volume does not differ between individuals with current AUD versus those with past AUD, supporting the hypothesis that reduced amygdala volume reflects a pre-existing risk factor for AUD rather than a consequence of personal exposure to alcohol $[23 \bullet \bullet]$.

Hippocampus One structure that has consistently shown the effects of alcohol neurotoxicity is the hippocampus [24]. Accordingly, it is of interest to determine if reduction in hippocampal volume might be associated with familial/genetic factors that preceded alcohol use. One study reported that high-risk male subjects had larger hippocampal volumes than did control male subjects [25]. Two studies with a small number of subjects found a reduction in hippocampal volume in adolescents with alcohol use disorders $[26,27]$ implying that personal alcohol exposures may lead to reduction in hippocampal volume. However, in a study that controlled for alcohol exposure, differences in hippocampal volume in association with risk status in male adolescents was not found though familial effects were seen for the right amygdala, which showed reduced volume [20].

Cerebellum Although classically considered a motor structure, the cerebellum has now been implicated in a number of cognitive processes, including those related to attention, working memory, learning, executive functioning, emotion, and affective states [28]. High-risk offspring from multiplex AD families show increased volume of the cerebellum and lesser decreases in cerebellar gray matter with age $[29,30]$, potentially indicating developmental delays in normative pruning of gray matter. Recent unpublished data (Hill et al., unpublished data) indicates that high-risk offspring show especially pronounced volumetric differences in the corpus and inferior posterior lobe of the cerebellum. These regions have been shown to be associated with higher-order cognitive and emotional processes [28]. 


\section{Connectomics and Risk for Alcohol Use and Related Substance Use Disorders}

The previous focus on specific brain regions in addiction are clearly important. However, recognizing that brain regions must function within the context of related brain regions, network analysis is essential for understanding the deficits that are associated with familial risk for substance use disorders. Moreover, separating causes from consequences requires a better understanding of how systems work together or fail to do so. Several brain networks have been described that appear to have important relevance to risk for developing an alcohol use or related substance use disorder. These include the frontoparietal control network [31], the cingulo-opercular control network (CON) [32], the salience processing network [33], the introspective socio-affective network (ISA), the corticostriatal network [34, 35], and the default mode network [36]. Each of these networks has been explored for a variety of neuropsychiatric disorders including substance use disorders. However, the techniques for uncovering resting state functional connectivity and structural connectivity continue to emerge, and exploration of these networks utilizing these tools is ongoing. Inclusion of all of the studies addressing the association between addiction and perturbations of these networks is beyond the scope of this review. However, a brief description of the networks and how they may provide insights into possible interventions or treatments will be offered. Regions associated with each network may be seen in Table 1 .

Fronto-Parietal Control Network Using resting state functional connectivity, Dosenbach and colleagues [31] analyzed their collected data using graph theory and hierarchical clustering techniques finding evidence that control regions of the brain are located in separate networks. The fronto-parietal network appears to be most concerned with initiated attentional control and with the task of integrating this control with regions that process performance feedback so that the individual has the opportunity to adjust his or her control settings. Because this network responds to feedback on a trial-by-trial basis, it may be relevant to decision-making activities that have been shown to be deficient in those with risk for substance use disorders $[37 \bullet \bullet]$.

Table 1 Major neural networks and associated brain regions with relevance to psychopathology

\begin{tabular}{|c|c|c|c|c|c|c|}
\hline Circuit & $\begin{array}{l}\text { Default } \\
\text { mode network }\end{array}$ & $\begin{array}{l}\text { Fronto-parietal } \\
\text { control network }\end{array}$ & $\begin{array}{l}\text { Cingulo-opercular } \\
\text { control (CON) } \\
\text { network }\end{array}$ & $\begin{array}{l}\text { Salience } \\
\text { processing } \\
\text { network }\end{array}$ & $\begin{array}{l}\text { Introspective } \\
\text { socio-affective } \\
\text { network }\end{array}$ & $\begin{array}{l}\text { Cortico-striatal } \\
\text { network }\end{array}$ \\
\hline Orbitofrontal cortex (OFC) & & & & $\mathrm{X}$ & & \\
\hline Medial superior frontal cortex (msFC) & & & $\mathrm{X}$ & & & \\
\hline Anterior prefrontal cortex (aPFC) & & & $\mathrm{X}$ & & & $\mathrm{X}$ \\
\hline Medial prefrontal cortex (mPFC) & $X$ & & & & & $\mathrm{X}$ \\
\hline Dorsomedial prefrontal cortex (dmPFC) & & & & & $\mathrm{X}$ & $\mathrm{X}$ \\
\hline Dorsolatral prefrontal cortex (DLPFC) & & $\mathrm{X}$ & & & & $\mathrm{X}$ \\
\hline Dorsal frontal cortex (dFC) & & $\mathrm{X}$ & & & & \\
\hline Dorsal anterior cingulate (dACC) & & & $\mathrm{X}$ & $X$ & & \\
\hline Middle cingulate cortex (mCC) & & $\mathrm{X}$ & & & & \\
\hline Posterior cingulate (PCC) & $\mathrm{X}$ & & & & & \\
\hline Precuneous (PrC) & $\mathrm{X}$ & $\mathrm{X}$ & & & $\mathrm{X}$ & \\
\hline Subgenual ACC (sdACC) & & & & & $\mathrm{X}$ & \\
\hline Anterior cingulate (aACC) & & & & & $\mathrm{X}$ & \\
\hline Intraparietal sulcus & & $\mathrm{X}$ & & & & \\
\hline Inferior parietal lobule (IPL) & & $\mathrm{X}$ & $\mathrm{X}$ & & & \\
\hline Posterior parietal cortex & $\mathrm{X}$ & & & & & \\
\hline Anterior insula (AI) & & & $\mathrm{X}$ & $\mathrm{X}$ & & \\
\hline Frontal operculum (FO) & & & $\mathrm{X}$ & & & \\
\hline Putamen & & & $\mathrm{X}$ & & & \\
\hline thalamus & & & $\mathrm{X}$ & & & \\
\hline Cerebellum & $\mathrm{X}$ & & $\mathrm{X}$ & & & \\
\hline amygdala & & & & & $\mathrm{X}$ & $\mathrm{X}$ \\
\hline Nucleus accumbens & & & & & & $\mathrm{X}$ \\
\hline Ventral tegmental area (VTA) & & & & & & $\mathrm{X}$ \\
\hline Caudate & & & & & & $\mathrm{X}$ \\
\hline
\end{tabular}


Cingulo-Opercular Control Network This network appears to be designed for maintenance of set across a series of trials so that the individual can maintain a bias that is based on previous experience within the entire task. The frontoparietal network and the cingulo-opercular network have been described as a dual network for top-down control [32]. Deficits in these two networks may also be related to deficits in decision making on the IGT observed in our lab and others.

Salience Processing Network The salience network has been described as one based in the dorsolateral prefrontal cortex (DLPFC) and orbitofrontal insular cortex with strong connections to subcortical and limbic areas that can be distinguished from an executive control network that links the parietal cortex with the dorsolateral frontal cortex [33]. This network is of particular relevance to findings from our lab showing reduction in both OFC $[15,16]$ and amygdala $[20,21 \bullet \cdot$ that are related to familial risk for alcohol dependence. In addition, we have observed that ratios of OFC to amygdala volume are associated with development of substance use disorder (Hill et al., unpublished data).

Introspective Socio-Affective Network This network has been identified through meta-analysis of studies using resting state functional magnetic resonance imaging (fMRI) in individuals with depression [38], and includes the OFC, dorsal anterior cingulate (dACC), and anterior insula. The function of this network appears to include social cognition and interpersonal expectations. This network may hold promise in studies of disorders involving alcohol and substance use, as frequently, there are co-occurring depressive symptomatology. Findings from our lab concerning social cognition suggest that the ISA network may have relevance to risk for developing an AUD or related SUD. In an fMRI study using the Eyes Task, which measures an individual's ability to read the other person's emotional state through facial expressions, we found that adolescent/young adult high-risk individuals had decreased BOLD activation in regions showing activation in controls [39]. Moreover, from a recent analysis of young adult offspring from families selected through women with alcohol dependence, we find whole brain reduction of gray matter in multiple brain regions but particularly in regions having to do with facial processing (insula, fusiform cortex), regions that also held up under further analysis using ROI measures (Hill et al., unpublished data). Additionally, using graph theory analysis of the same young adult sample, we find that lowrisk control offspring showed greater connectivity than highrisk offspring between the middle temporal gyrus and the anterior cingulate, and between the fusiform gyrus and superior temporal and middle temporal gyri.

The Cortico-Striatal Network This network includes connections between the DLPFC and the caudate nucleus along with the nucleus accumbens, ventral tegmental area, prefrontal cortex, and amygdala [35, 40]. This network has a long history in the addiction field and appears to continue to hold promise for understanding the underpinnings of addictive behavior.

The Default Mode Network The identification of this network through temporal correlations of low-frequency fluctuations between brain regions at rest has been considered a major breakthrough in our understanding of how the brain responds to internal and external cues shifting from the default mode to a mode relevant to task requirements. Inability to rapidly accomplish this has been associated with a variety of neuropsychiatric disorders. Identification of the DMN was first shown in Alzheimer's disease [36] and may have implications for how high-risk offspring engage in task related activity, an area where they have been shown to have deficits.

\section{Electrophysiological Characteristics of High-Risk Offspring}

During childhood and adolescence, morphological changes in brain development appear to be accompanied by changes in electrophysiological neural activity [41]. Studies examining electroencephalographic (EEG) or event-related potential (ERP) activity during cognitive tasks have documented differing patterns of activity in children and adolescents from alcoholic families in comparison to controls. The ERP waveform is typically described in terms of components that are defined by specific latency windows (i.e., P100, N100, P200, N200, and P300). Components having negative polarity include N100 and N200, while positive polarity is seen for $\mathrm{P} 100, \mathrm{P} 200$, and $\mathrm{P} 300$.

\section{P300: an Endophenotype of Substance Use Disorder Risk}

Reduction in P300 amplitude was first identified in association with schizophrenia [42]. The relevance of P300 in other psychiatric disorders, particularly those involving externalizing behaviors (e.g., conduct problems) that commonly precede and predict AUD/SUD, has been now well-documented. As noted previously, one requirement that a biomarker is a potentially useful endophenotype is whether it is heritable. Data from a large community sample of adolescent twins and their parents indicate that P300 amplitude is heritable [43••]. Previous biometrical modeling of sib-sib, parentchild, and grandparent-grandchild similarity showed similar heritability rates in both high- and low-risk samples [44]. Additionally, P300 appears to be independent of disease state, as numerous cross-sectional studies have demonstrated that P300 amplitude varies in individuals with a family history in comparison to controls [45].

The historical antecedent for the P300 ERP component emerging as a potential endophenotype of risk for alcohol use 
disorder occurred over three decades ago when Begleiter and colleagues [46] observed that sons of alcohol dependent men had lower amplitude P300 than did sons of control fathers. Subsequently, many laboratories confirmed these results in both child/adolescent offspring from families with alcohol dependence [47]. The P300 component is a large positive waveform that reaches its peak approximately $300 \mathrm{~ms}$ after stimulus onset. The amplitude of the P300 component is typically defined by the difference between the prestimulus baseline voltage and the largest peak of the component within a predefined latency window depending on the modality (visual or auditory). Although P300 can be elicited with the subject being asked to perform a task [48], typically subjects are instructed to respond to target stimulus and ignore an alternate nontarget stimulus. Consequently, performance of such odd-ball tasks requires the subject to engage in working memory task in order to correctly respond by pressing a button, counting targets, or similar measures of behavioral performance.

P300, Childhood Psychopathology, and Young Adult Outcome Many psychiatric disorders show abnormalities of neurodevelopment. Therefore, the utility of P300 as a biomarker of psychiatric illness may have to do with its relationship to neurodevelopmental processes. Utilizing data acquired from approximately annual, repeated P300 assessments, Hill and colleagues [49] performed growth curve modeling for 635 P300 assessments in which two thirds of the sample were tested five or more times. This analysis was the first to show that P300 amplitude was not a static characteristic of the individual but varied across development. Additionally, this analysis revealed that high-risk offspring from multiplex, alcoholdependent families show atypical developmental trajectories of P300 amplitude, with slower rate of change with age.

Using data acquired for offspring seen through childhood and adolescence, mixture analysis was conducted on the trajectories of P300 development resulting from these repeated measures [50]. Using the best fit model, three trajectory classes were found each with associated risks for developing psychopathogy. There was a two-fold increase in the number of children at high familial risk for alcohol dependence due to their family background in the Class 3 pattern characterized by a slower and lower P300 amplitude trajectory [50]. Additionally, among the high-risk children in Class 3 , significantly more of these children had a lifetime diagnosis of psychopathology. These early results supported the continued use of P300 as a potential biomarker of familial risk and childhood diagnosis and suggested that $\mathrm{P} 300$ amplitude might be a useful biomarker for SUD outcome in young adulthood and beyond.

\section{P300 and Genetic Variation}

A number of candidate genes have been investigated with respect to their potential role in the generation of $\mathrm{P} 300$, some showing better replication than others [43••]. Studies showing the greatest consistency concern dopamine $[51,52 \bullet, 53]$ and the muscarinic acetylcholine receptor $[54,55 \bullet \cdot$. Further exploration of candidate genes may be warranted where withinfamily variation in genetic polymorphisms are seen in association with both alcohol dependence and P300 variation, as was recently found for the ACN9 gene [56].

P300: An Indicator of Future Substance Use Disorder P300 amplitude at age 9 was associated with development of substance use disorders 11 years later in young adulthood [57]. Other follow-up studies have found that P300 assessed at one point in time predicts substance use disorders at a later point in time. Habeych and colleagues [58] reported that P300 amplitude predicted SUD outcome at 7 years as has Iacono and colleagues[59] in a 3-year follow-up. The origin of this relationship between P300 amplitude and outcome may derive from its association with a wide variety of externalizing and deviant behaviors that have now been shown to be associated with lower amplitude of P300 [59, 60].

\section{Postural Control in High-Risk Offspring}

High-risk offspring demonstrate less postural control and greater body sway, as measured on a computerized movement platform [61]. Longitudinal follow-ups of these offspring indicate that they show lesser decrease in body sway with age, likely reflecting a developmental delay [62]. Additionally, increased postural sway during adolescence is a particularly salient predictor of subsequent substance use outcomes among high-risk individuals [57]. In alcohol-dependent adults, postural sway is associated with abnormal cerebellar morphology [63], and the developmental delays in postural control in high-risk offspring may relate to concurrently observed abnormal trajectories of cerebellar development $[29,30]$. In addition, when childhood P300 amplitude was combined with assessment of body sway as measured by a computerized movement platform, individuals in the lowest tercile for P300 amplitude and the highest tercile for body sway had an eightfold increase in risk for developing SUD by young adulthood [57].

\section{Psychological Characteristics of High-Risk Offspring}

Premorbid behavioral and cognitive risk factors have also been observed in children and adolescents with a family history of alcoholism that are predictive of subsequent substance use disorders [64]. Deficits in response inhibition, cognitive control, and emotion regulation are commonly observed in 
high-risk individuals. Externalizing behaviors, including oppositionality, hyperactivity, impulsivity, inattention, and sensation seeking are observed in high-risk offspring and are predictors of alcohol and drug use problems [64]. Similarly, high-risk offspring demonstrate increased risk for externalizing disorders in childhood and adolescence, including attention deficit hyperactivity disorder, oppositional defiant disorder, and conduct disorder [65, 66]. High-risk offspring also have a higher incidence of internalizing psychopathology and demonstrate anxiety and stress-reactive personality traits such as harm avoidance, low self-esteem, negative affectivity, and impaired emotion regulation [64-66]. Recent research on psychological predictors of alcohol use disorders in high-risk offspring has focused on impaired reward sensitivity and decision making, as well as deficits in social cognition.

\section{Reward Sensitivity and Maladaptive Decision} Making Adolescents and young adults with a family history of AUD have been characterized as having atypical reward sensitivity that affects decision-making abilities. On the Iowa Gambling Task (IGT), a measure of decision making in which successful performance depends on selecting stimuli (i.e., cards) associated with lower short-term, but higher long-term, rewards [18], high-risk offspring show greater attention to monetary gains as well as atypical neural activation in the anterior cingulate and caudate during this task [67, 68]. Recent data from our laboratory indicate that maladaptive decision making may be an important precursor to substance use disorders in high-risk adolescents and young adults [37••]. Individuals from multiplex, alcohol-dependent families showed poorer overall performance on the IGT, and especially poor performance on the final trial block, indicating a failure to improve decision making with previous experience. Importantly, survival analyses indicated that poor performance on the final trial block was significantly associated with substance use disorders in young adulthood [37•0]. Previous research has implicated the prefrontal cortex in adaptive decision making, suggesting that the observed decision-making deficits in high-risk offspring [37०•] may be partially related to observed morphological alterations in the orbitofrontal cortex $[15,16]$.

Social Cognition Preliminary evidence suggests that deficits in social cognition may be a premorbid risk factor for AUD. Highrisk offspring show atypical neural activation when viewing emotional faces $[39,69 \bullet, 70 \bullet, 71]$, and neural activation to emotionally valenced words among high-risk offspring is related to problem drinking in adolescence [72]. Several aspects of social cognition have been shown to relate to the onset and course of alcohol use disorders, such that poorer social cognition predicts early alcohol and drug use, greater severity of AUD, and poorer treatment outcomes [73-75]. However, most research on social cognition in high-risk youth has focused on fMRI paradigms involving passive viewing of emotional faces, and future research with an expanded range of ecologically valid experimental paradigms is needed to determine the extent of social cognition deficits in high-risk offspring.

\section{Conclusions}

Alcohol use disorders are complex conditions that are influenced by genetic and environmental factors and their interaction. The search for genes that may increase susceptibility to alcohol dependence has been greatly facilitated by the recognition that intermediate phenotypes, sometimes referred to as endophenotypes, may be closer to the genetic variation than is the more complex alcohol dependence phenotype [3]. Given that alcohol use disorders run in families, research examining substance-naive, first-degree relatives of individuals with alcohol use disorders has elucidated a number of biological and psychological characteristics that may reflect premorbid risk factors for AUD. Additionally, longitudinal studies following these offspring have allowed for the determination of risk and resilience factors among these high-risk offspring.

Research among high-risk offspring has indicated that these individuals demonstrate atypical morphology of neural structures associated with executive control (orbitofrontal cortex, cerebellum) and affective regulation (amygdala). Highrisk offspring also show developmental delays in electrophysiological activity and postural control. These biological risk factors may underlie observed psychological and cognitive deficits associated with high-risk status, including decreased behavioral inhibition, increased reward sensitivity, impaired decision making, and poor social cognition. Variation among high-risk offspring also shows that more severe deficits in several of these domains are associated with greater risk for subsequent alcohol and drug use problems.

Traits associated with risk for AUD often converge with observed deficits in alcohol-dependent individuals, indicating that the behavioral phenotype of AUD likely reflects a complex interaction of premorbid structural and functional neural abnormalities as well as the neurotoxic effects of alcohol and other drugs of abuse on the brain. Future longitudinal research is needed to disentangle cause from consequence in alcohol use disorders, as well as to determine which characteristics of highrisk offspring may be most efficacious to target in behavioral intervention efforts. In addition, an important area for further study is the merging of genetic methodologies with neuroimaging approaches within samples of high- and low-risk individuals to elucidate biological pathways that may inform medication development for the treatment of alcohol use disorders.

\section{Compliance with Ethics Guidelines}

Conflict of Interest Shirley Hill and Jessica O'Brien declare no conflict of interest. 
Human and Animal Rights and Informed Consent This article does not contain any studies with human or animal subjects performed by any of the authors.

\section{References}

Papers of particular interest, published recently, have been highlighted as:

- Of importance

•- Of major importance

1. Hasin DS, Stinson FS, Ogburn E, Grant BF. Prevalence, correlates, disability, and comorbidity of DSM-IV alcohol abuse and dependence in the United States: results from the National Epidemiologic Survey on Alcohol and Related Conditions. Arch Gen Psychiatry. 2007;64(7):830-42.

2. Verhulst B, Neale MC, Kendler KS. The heritability of alcohol use disorders: a metal-analysis of twin and adoption studies. Psychol Med. 2014. doi:10.1017/S0033291714002165.

3. Hill SY. Neural plasticity, human genetics, and risk for alcohol dependence. Int Rev Neurobiol. 2010;91:53-94. doi:10.1016/ S0074-7742(10)91003-9.

4. Gottesman II, Gould TD. The endophenotype concept in psychiatry: etymology and strategic intentions. Am J Psychiatry. 2003;160(4):636-45.

5. Dick DM, Bierut L, Hinrichs A, Fox L, Bucholz KK, Kramer J, et al. The role of GABRA2 in risk for conduct disorder and alcohol and drug dependence across developmental stages. Behav Genet. 2006;36(4):577-90.

6. Casey BJ, Tottenham N, Liston C, Durston S. Imaging the developing brain: what have we learned about cognitive development? Trends Cogn Sci. 2005;9(3):104-10.

7. Crone EA, Dahl RE. Understanding adolescence as a period of social-affective engagement and goal flexibility. Nat Rev Neurosci. 2012;13(9):636-50

8. Gogtay N, Giedd JN, Lusk L, Hayashi KM, Greenstein D, Vaituzis $\mathrm{AC}$, et al. Dynamic mapping of human cortical development during childhood through early adulthood. Proc Natl Acad Sci U S A. 2004;101(21):8174-9.

9. Giedd JN. Structural magnetic resonance imaging of the adolescent brain. Ann N Y Acad Sci. 2004;1021:77-85.

10. Giedd JN, Raznahan A, Alexander-Bloch A, Schmitt E, Gogtay N, Rapoport JL. Child psychiatry branch of the national institute of mental health longitudinal structural magnetic resonance imaging study of human brain development. Neuropsychopharmacology. 2015;40(1):43-9. This manuscript provides a comprehensive review of normal brain development and outlines challenges for future studies.

11. Ostby Y, Tamnes CK, Fjell AM, Westlye LT, Due-Tønnessen P, Walhovd KB. Heterogeneity in subcortical brain development: a structural magnetic resonance imaging study of brain maturation from 8 to 30 years. J Neurosci. 2009;29(38):11772-82.

12. Pfefferbaum A, Sullivan EV, Carmelli D. Morphological changes in aging brain structures are differentially affected by time-linked environmental influences despite strong genetic stability. Neurobiol Aging. 2004;25(2):175-83.

13. Peper JS, Brouwer RM, Boomsma DI, Kahn RS, Hulshoff Pol HE. Genetic influences on human brain structure: a review of brain imaging studies in twins. Hum Brain Mapp. 2007;28(6):464-73.

14. Goldstein RZ, Volkow ND. Dysfunction of the prefrontal cortex in addiction: neuroimaging findings and clinical implications. Nat Rev Neurosci. 2011;12(11):652-69.
15. Hill SY, Tessner K, Wang S, Carter H, McDermott M. Temperament at 5 years of age predicts amygdala and orbitofrontal volume in the right hemisphere in adolescence. Psychiatry Res. 2010;182(1):14-21.

16. Hill SY, Wang S, Kostelnik B, Carter H, Holmes B, McDermott M, et al. Disruption of orbitofrontal cortex laterality in offspring from multiplex alcohol dependence families. Biol Psychiatry. 2009;65(2):129-36

17. Tranel D, Bechara A, Denburg NL. Asymmetric functional roles of right and left ventromedial prefrontal cortices in social conduct, decision-making, and emotional processing. Cortex. 2002;38(4): 589-612.

18. Bechara A, Damasio AR, Damasio H, Anderson SW. Insensitivity to future consequences following damage to human prefrontal cortex. Cognition. 1994;50(1-3):7-15.

19. Jasinska AJ, Stein EA, Kaiser J, Naumer MJ, Yalachkov Y. Factors modulating neural reactivity to drug cues in addiction: a survey of human neuroimaging studies. Neurosci Biobehav Rev. 2014;38:116.

20. Hill SY, De Bellis MD, Keshavan MS, Lowers L, Shen S, Hall J, et al. Right amygdala volume in adolescent and young adult offspring from families at high risk for developing alcoholism. Biol Psychiatry. 2001;49(11):894-905.

21.• Hill SY, Wang S, Carter H, McDermott MD, Zezza N, Stiffler S. Amygdala volume in offspring from multiplex for alcohol dependence families: the moderating influence of childhood environment and 5-HTTLPR variation. J Alcohol Drug Depend. 2013;Supp 1: 001. This study showed the positive impact that family environment can have on amygdala volume that is associated with 5HTTLPR variation but only in offspring from control families.

22. Benegal V, Antony G, Venkatasubramanian G, Jayakumar PN. Gray matter volume abnormalities and externalizing symptoms in subjects at high risk for alcohol dependence. Addict Biol. 2007;12(1):122-32.

23.• Dager AD, McKay DR, Kent Jr JW, Curran JE, Knowles E, Sprooten E, et al. Shared genetic factors influence amygdala volumes and risk for alcoholism. Neuropsychopharmacology. 2015;40(2):412-20. This study confirmed that amygdala volume is reduced in offspring at high risk for developing alcohol dependence due to their familial/genetic background.

24. Sullivan EV, Marsh L, Mathalon DH, Lim KO, Pfefferbaum A. Anterior hippocampal volume deficits in nonamnesic, aging chronic alcoholics. Alcohol Clin Exp Res. 1995;19(1):110-22.

25. Hanson KL, Medina KL, Nagel BJ, Spadoni AD, Gorlick A, Tapert SF. Hippocampal volumes in adolescents with and without a family history of alcoholism. Am J Drug Alcohol Abuse. 2010;36(3):1617.

26. De Bellis MD, Clark DB, Beers SR, Soloff PH, Boring AM, Hall J, et al. Hippocampal volume in adolescent-onset alcohol use disorders. Am J Psychiatry. 2000;157(5):737-44.

27. Nagel BJ, Schweinsburg AD, Phan V, Tapert SF. Reduced hippocampal volume among adolescents with alcohol use disorders without psychiatric comorbidity. Psychiatry Res. 2005;139(3):181-90.

28. O'Halloran CJ, Kinsella GJ, Storey E. The cerebellum and neuropsychological functioning: a critical review. J Clin Exp Neuropsychol. 2012;34(1):35-56.

29. Hill SY, Muddasani S, Prasad K, Nutche J, Steinhauer SR, Scanlon $\mathrm{J}$, et al. Cerebellar volume in offspring from multiplex alcohol dependence families. Biol Psychiatry. 2007;61(1):41-7.

30. Hill SY, Wang S, Carter $\mathrm{H}$, Tessner $\mathrm{K}$, Holmes B, McDermott $\mathrm{M}$, et al. Cerebellum volume in high-risk offspring from multiplex alcohol dependence families: association with allelic variation in GABRA2 and BDNF. Psychiatry Res. 2011;194(3):304-13.

31. Dosenbach NU, Fair DA, Miezin FM, Cohen AL, Wenger KK, Dosenbach RA, et al. Distinct brain networks for adaptive and 
stable task control in humans. Proc Natl Acad Sci U S A. 2007;104(26):11073-8.

32. Dosenbach NU, Fair DA, Cohen AL, Schlaggar BL, Petersen SE. A dual-networks architecture of top-down control. Trends Cogn Sci. 2008;12(3):99-105.

33. Seeley WW, Menon V, Schatzberg AF, Keller J, Glover GH, Kenna $\mathrm{H}$, et al. Dissociable intrinsic connectivity networks for salience processing and executive control. J Neurosci. 2007;27(9):2349-56.

34. Tu PC, Hsieh JC, Li CT, Bai YM, Su TP. Cortico-striatal disconnection within the cingulo-opercular network in schizophrenia revealed by intrinsic functional connectivity analysis: a resting fMRI study. Neuroimage. 2012;59(1):238-47.

35. Kalivas PW. The glutamate homeostasis hypothesis of addiction. Nat Rev Neurosci. 2009;10(8):561-72.

36. Greicius MD, Srivastava G, Reiss AL, Menon V. Default-mode network activity distinguishes Alzheimer's disease from healthy aging: evidence from functional MRI. Proc Natl Acad Sci U S A. 2004;101(13):4637-42.

37.• O'Brien JW, Lichenstein SD, Hill SY. Maladaptive decision making and substance use outcomes in high risk individuals: preliminary evidence for the role of 5-HTTLPR variation. J Stud Alcohol Drugs. 2014;75(4):643-52. This is the first study to show that both familial risk for SUD and 5-HTTLPR variation impact performance on the IGT. Poor IGT performance was associated with earlier onset of SUD, suggesting that high-risk individuals who fail to appropriately attend to long term costs and benefits during a decision-making task are especially at risk for developing SUD in adolescence and young adulthood.

38. Schilbach L, Müller VI, Hoffstaedter F, Clos M, Goya-Maldonado $\mathrm{R}$, Gruber $\mathrm{O}$, et al. Meta-analytically informed network analysis of resting state fMRI reveals hyperconnectivity in an introspective socio-affective network in depression. PLoS One. 2014;9(4): e94973.

39. Hill SY, Kostelnik B, Holmes B, Goradia D, McDermott M, Diwadkar V, et al. fMRI BOLD response to the eyes task in offspring from multiplex alcohol dependence families. Alcohol Clin Exp Res. 2007;31(12):2028-35.

40. Leh SE, Ptito A, Chakravarty MM, Strafella AP. Fronto-striatal connections in the human brain: a probabilistic diffusion tractography study. Neurosci Lett. 2007;419(2):113-8.

41. Segalowitz SJ, Santesso DL, Jetha MK. Electrophysiological changes during adolescence: a review. Brain Cogn. 2010;72(1): 86-100.

42. Steinhauer S, Zubin J. Vulnerability to schizophrenia: Information processing in the pupil and event-related potential. In: Hanin I, Usdin E, editors. Biological markers in psychiatry and neurology. Oxford: Pergamon Press; 1982. p. 371-85.

43.• Malone SM, Vaidyanathan U, Basu S, Miller MB, McGue M, Iacono WG. Heritability and molecular-genetic basis of the P3 event-related brain potential: a genome-wide association study. Psychophysiology. 2014;51(12):1246-58. This manuscript provides a review of evidence in support of $P 300$ as a heritable phenotype that has been related to genetic polymorphism involved in neurotransmitter functioning.

44. Hill SY, Yuan H, Locke J. Path analysis of P300 amplitude of individuals from families at high and low risk for developing alcoholism. Biol Psychiatry. 1999;45(3):346-59.

45. Euser AS, Arends LR, Evans BE, Greaves-Lord K, Huizink AC, Franken IH. The P300 event-related brain potential as a neurobiological endophenotype for substance use disorders: a meta-analytic investigation. Neurosci Biobehav Rev. 2012;36(1):572-603.

46. Begleiter H, Porjesz B, Bihari B, Kissin B. Event-related brain potentials in boys at risk for alcoholism. Science. 1984;225(4669):1493-6.
47. Hill SY, Steinhauer S, Park J, Zubin J. Event-related potential characteristics in children of alcoholics from high density families. Alcohol Clin Exp Res. 1990;14(1):6-16.

48. Polich J. Overview of P3a and P3b. In: Polich J, editor. Detection of change: event-related potential and fMRI findings. Norwell: Kluwer Academic Press; 2003. p. 83-98.

49. Hill SY, Shen S, Locke J, Steinhauer SR, Konicky C, Lowers L, et al. Developmental delay in P300 production in children at high risk for developing alcohol-related disorders. Biol Psychiatry. 1999;46(7):970-81.

50. Hill SY, Shen S. Neurodevelopmental patterns of visual P3b in association with familial risk for alcohol dependence and childhood diagnosis. Biol Psychiatry. 2002;51(8):621-31.

51. Hill SY, Locke J, Zezza N, Kaplan B, Neiswanger K, Steinhauer SR, et al. Genetic association between reduced P300 amplitude and the DRD2 dopamine receptor A1 allele in children at high risk for alcoholism. Biol Psychiatry. 1998;43(1):40-51.

52. Heitland I, Kenemans JL, Oosting RS, Baas JM, Böcker KB. Auditory event-related potentials ( $\mathrm{P} 3 \mathrm{a}, \mathrm{P} 3 \mathrm{~b})$ and genetic variants within the dopamine and serotonin system in healthy females. Behav Brain Res. 2013;249:55-64. This manuscript illustrates the influence of genetic variation in two genetic variants on P300 amplitude.

53. Neuhaus AH, Goldberg TE, Hassoun Y, Bates JA, Nassauer KW, Sevy S, et al. Acute dopamine depletion with branched chain amino acids decreases auditory top-down event-related potentials in healthy subjects. Schizophr Res. 2009;111(1-3):167-73.

54. Jones KA, Porjesz B, Almasy L, Bierut L, Dick D, Goate A, et al. A cholinergic receptor gene (CHRM2) affects event-related oscillations. Behav Genet. 2006;36(5):627-39.

55.• Hill SY, Jones BL, Holmes B, Steinhauer SR, Zezza N, Stiffler S. Cholinergic receptor gene (CHRM2) variation and familial loading for alcohol dependence predict childhood developmental trajectories of P300. Psychiatry Res. 2013;209(3):504-11. This study shows the influence of the cholinergic receptor gene (CHRM2) on trajectories of P300 during childhood. Importantly, CHRM2 variation in the COGA sample is in accord with these findings (54).

56. Hill SY, Jones BL, Zezza N, Stiffler S. ACN9 and alcohol dependence: family-based association analysis in multiplex alcohol dependence families. Am J Med Genet B Neuropsychiatr Genet. 2015; 168B(3):179-187.

57. Hill SY, Steinhauer SR, Locke-Wellman J, Ulrich R. Childhood risk factors for young adult substance dependence outcome in offspring from multiplex alcohol dependence families: a prospective study. Biol Psychiatry. 2009;66(8):750-7.

58. Habeych ME, Charles PJ, Sclabassi RJ, Kirisci L, Tarter RE. Direct and mediated associations between P300 amplitude in childhood and substance use disorders outcome in young adulthood. Biol Psychiatry. 2005;57(1):76-82.

59. Iacono WG, Carlson SR, Malone SM, McGue M. P3 event-related potential amplitude and the risk for disinhibitory disorders in adolescent boys. Arch Gen Psychiatry. 2002;59(8):750-7.

60. McGue M, Iacono WG, Legrand LN, Malone S, Elkins I. Origins and consequences of age at first drink. I. Associations with substance-use disorders, disinhibitory behavior and psychopathology, and P3 amplitude. Alcohol Clin Exp Res. 2001;25(8):115665 .

61. Hill SY, Steinhauer S. Postural sway in children from pedigrees exhibiting a high density of alcoholism. Biol Psychiatry. 1993;33(5):313-25.

62. Hill SY, Shen S, Locke J, Lowers L, Steinhauer S, Konicky C. Developmental changes in postural sway in children at high and low risk for developing alcohol-related disorders. Biol Psychiatry. 2000;47(6):501-11. 
63. Sullivan EV, Rose J, Pfefferbaum A. Effect of vision, touch and stance on cerebellar vermian-related sway and tremor: a quantitative physiological and MRI study. Cereb Cortex. 2006;16(8):1077-86.

64. Tessner KD, Hill SY. Neural circuitry associated with risk for alcohol use disorders. Neuropsychol Rev. 2010;20(1):1-20.

65. Hill SY, Shen S, Lowers L, Locke-Wellman J, Matthews AG, McDermott M. Psychopathology in offspring from multiplex alcohol dependence families with and without parental alcohol dependence: a prospective study during childhood and adolescence. Psychiatry Res. 2008;160(2):155-66.

66. Hill SY, Tessner KD, McDermott MD. Psychopathology in offspring from families of alcohol dependent female probands: a prospective study. J Psychiatr Res. 2011;45(3):285-94.

67. Lovallo WR, Yechiam E, Sorocco KH, Vincent AS, Collins FL. Working memory and decision-making biases in young adults with a family history of alcoholism: studies from the Oklahoma family health patterns project. Alcohol Clin Exp Res. 2006;30(5):763-73.

68. Acheson A, Robinson JL, Glahn DC, Lovallo WR, Fox PT. Differential activation of the anterior cingulate cortex and caudate nucleus during a gambling simulation in persons with a family history of alcoholism: studies from the Oklahoma Family Health Patterns Project. Drug Alcohol Depend. 2009;100(1-2):17-23.

69. Hulvershorn LA, Finn P, Hummer TA, Leibenluft E, Ball B, Gichina V, et al. Cortical activation deficits during facial emotion processing in youth at high risk for the development of substance use disorders. Drug Alcohol Depend. 2013;131(3):230-7. This study illustrates the importance of emotional processing as a risk factor for addiction.

70. Peraza J, Cservenka A, Herting MM, Nagel BJ. Atypical parietal lobe activity to subliminal faces in youth with a family history of alcoholism. Am J Drug Alcohol Abuse. 2015;41:139-45. doi:10. 3109/00952990.2014.953251. This study finds facial processing associated with familial/genetic risk, supporting references $(39,69)$.

71. Glahn DC, Lovallo WR, Fox PT. Reduced amygdala activation in young adults at high risk of alcoholism: studies from the Oklahoma family health patterns project. Biol Psychiatry. 2007;61(11):13069.

72. Heitzeg MM, Nigg JT, Yau WY, Zubieta JK, Zucker RA. Affective circuitry and risk for alcoholism in late adolescence: differences in frontostriatal responses between vulnerable and resilient children of alcoholic parents. Alcohol Clin Exp Res. 2008;32(3):414-26.

73. Thorberg FA, Young RM, Sullivan KA, Lyvers M. Alexithymia and alcohol use disorders: a critical review. Addict Behav. 2009;34(3):237-45.

74. Uekermann J, Daum I. Social cognition in alcoholism: a link to prefrontal cortex dysfunction? Addiction. 2008;103(5):726-35.

75. Thoma P, Friedmann C, Suchan B. Empathy and social problem solving in alcohol dependence, mood disorders and selected personality disorders. Neurosci Biobehav Rev. 2013;37(3):448-70. 